\title{
Ventral Prefrontal Cortex Is Not Essential for Working Memory
}

\author{
Matthew F. S. Rushworth, ${ }^{1}$ Philip D. Nixon, ${ }^{1}$ Madeline J. Eacott, ${ }^{2}$ and Richard E. Passingham ${ }^{1}$ \\ ${ }^{1}$ Department of Experimental Psychology, University of Oxford, Oxford OX1 3UD, United Kingdom, and ${ }^{2}$ Department of \\ Psychology, University of Durham Durham, DH1 3LE, United Kingdom
}

It is widely held that the prefrontal cortex is important for working memory. It has been suggested that the inferior convexity (IC) may play a special role in working memory for form and color (Wilson et al., 1993). We have therefore assessed the ability of monkeys with IC lesions to perform visual pattern association tasks and color-matching tasks, both with and without delay. In experiment 1 , six monkeys were trained on a visual association task with delays of up to $2 \mathrm{sec}$. Conservative IC lesions that removed lateral area 47/12 in three animals had no effect on the task. Further experiments showed that these lesions had no effect on the postoperative new learning of a color-matching task with delays of up to 2 sec or versions of the visual association task involving delays of up to $8 \mathrm{sec}$. In experiment 2, larger lesions of both areas 47/12 and 45A were made in the three control animals. This lesion caused a profound deficit in the ability to relearn simultaneous color matching, but subsequent matching with delays of up to $8 \mathrm{sec}$ was clearly unimpaired. We suggest that the IC may be more important for stimulus selection and attention as opposed to working memory.

Key words: prefrontal cortex; working memory; inferior convexity; area 12; area 47; object vision; positron emission tomography
It is widely held that the prefrontal cortex is involved in working memory (Goldman-Rakic, 1987, 1996; Funahashi and Kubota, 1994). In animal-based research, working memory refers to the ability to hold information "on-line" to guide behavior in the absence of explicit cues for action selection. In tests of working memory, unlike tests of associative memory, the same nonunique stimuli are used trial after trial in delayed response (DR) and delayed alternation (DA) tasks; the tests critically require that information held in memory is changed and updated from trial to trial.

It has recently been proposed that there is a parallel organization such that dorsal and ventral parts of the prefrontal cortex are concerned with working memory for spatial and object or form information, respectively. This proposal is based on the delaydependent selectivities of single units in the sulcus principalis and the inferior convexity (IC), respectively, in DR tasks (Wilson et al., 1993). A large number of human imaging studies have been interpreted in terms of this dichotomy (for review, see GoldmanRakic, 1996).

The fact that single units in monkeys or populations of cells in imaging studies are more active during delays need not imply that the basic function of the area is to bridge those delays. An analogy can be made with the dorsal premotor cortex, in which both single-unit studies and imaging studies have demonstrated activity related to delays that precede movement execution (Wise and Mauritz, 1985; Kalaska and Crammond, 1995; Deiber et al., 1996). The premotor cortex is not, however, regarded as a working memory structure; it is better characterized as having a role in movement selection. Its removal disrupts movement selection even in the absence of a delay before execution (Passingham, 1988). The activity of premotor cortex cells during delays might be

Received Oct. 18, 1996; revised March 18, 1997; accepted March 27, 1997.

This work was supported by a Wellcome program grant. We thank M. Brown and D. French for technical assistance and C. Healey Yorke for histological processing. Correspondence should be addressed to Matthew Rushworth, Department of Experimental Psychology, University of Oxford, South Parks Road, Oxford OX1 3UD, UK.

Copyright (C) 1997 Society for Neuroscience $\quad 0270-6474 / 97 / 174829-10 \$ 05.00 / 0$ better interpreted as corresponding to the initial stages of a movement selection that precede movement execution. The premotor cortex may be able to hold movements in memory, but it is clear that its function is not restricted to memory.

The results are different for the sulcus principalis (area 46). It is clear that it plays some role in spatial working memory. Lesions disrupt spatial DR and DA (Mishkin, 1957; Butters and Pandya, 1969; Passingham, 1985), and there is no effect unless the task has both delay (Goldman and Rosvold, 1970; Passingham, 1985; Funahashi et al., 1993) and spatial components (Passingham, 1975; Mishkin and Manning, 1978).

Passingham (1993), however, has pointed out that it is not clear whether IC lesions only cause delay-related impairments. Delay impairments have been recorded without any attempt first to retrain the animals on simultaneous versions of the tasks. It is therefore possible that poor performance was not specifically related to the memory demands of the task; it may be attributable to relearning other aspects of the task. There are also impairments on tasks, such as go-no go, with no delay component.

The present study examines the effect of IC lesions on visual pattern association and color-matching tasks. In each case stimuli were not trial unique. Postoperatively, we first tested the animals' relearning of nondelay versions of the task and assessed performance with delays only after they had reached criterion without delay. The lesions in the first experiment were conservative and focused on the anterior part of the IC, the lateral part of area 47/12 (Petrides and Pandya, 1994; Fig. 1). We thought it important not to invade the prearcuate area, because lesions there cause impairments on visual associative tasks even without delays (Halsband and Passingham, 1985; Petrides, 1987). In the second experiment the lesion was extended both ventromedially and caudally into area 45A (Fig. 1).

\section{MATERIALS AND METHODS}

\section{Subjects}

Six rhesus macaques (Macaca mulatta) were used, aged between 2 and 4 years and weighing between 3 and $4 \mathrm{~kg}$. The guidelines in Principles of 

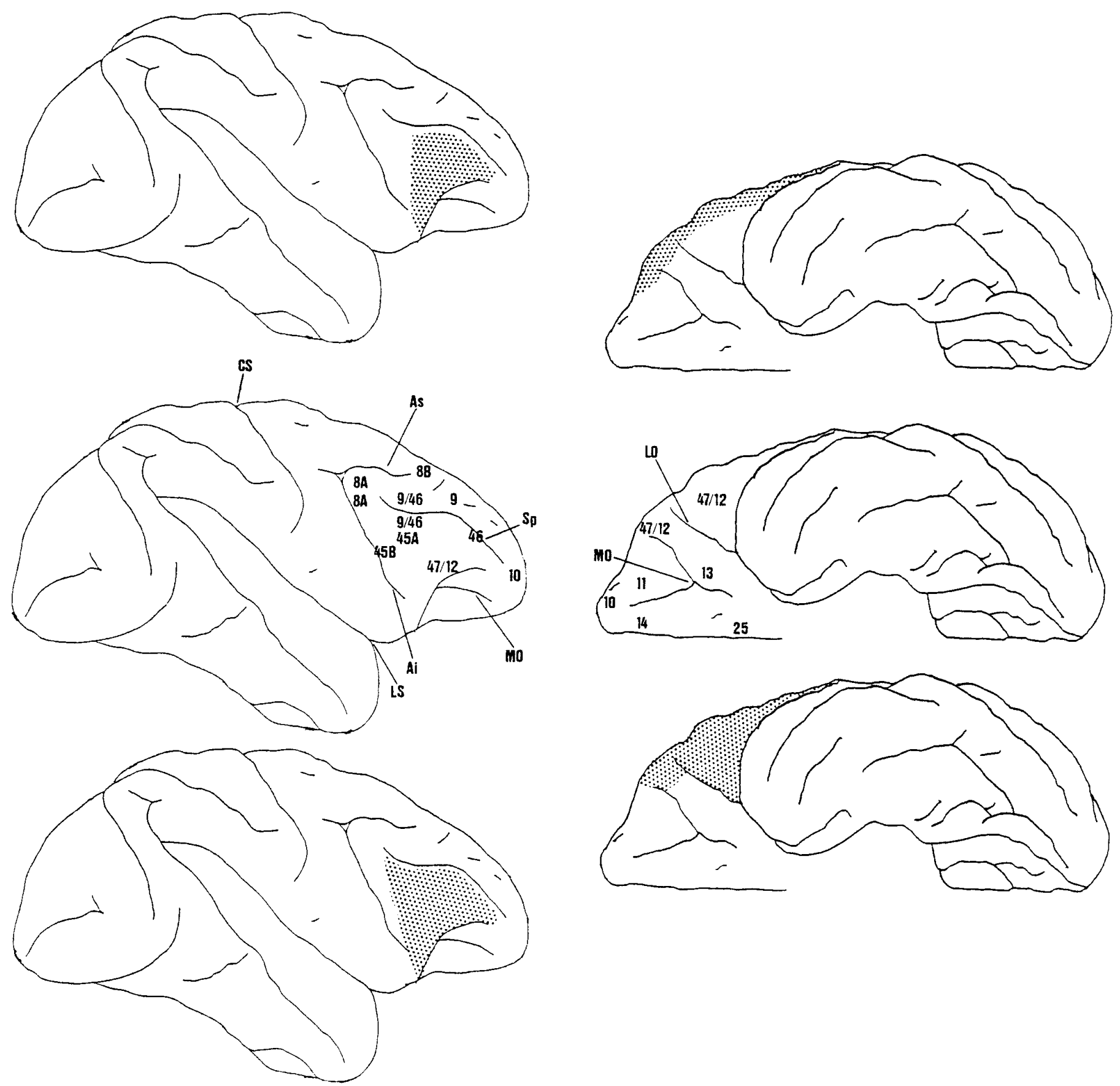

Figure 1. Lateral and ventral views of the intended lesions in the two experiments. The center diagrams show the parcellation of prefrontal anatomical areas in the macaque monkey proposed by Petrides and Pandya (1994). Area 47/12 is situated on the anterior surface of the lateral IC (left); ventromedially it extends as far as the lateral orbital sulcus $(L O)$ on the posterior orbital surface and as far as the lateral bifurcation of the medial orbital sulcus $(M O)$ on the more anterior orbital surface (right). The lateral and orbital parts of 47/12 have been labeled 47/12l and 47/12o (Petrides and Pandya, 1994; or 121 and 12o, Carmicheal and Price, 1994). Area 45A is situated more posterior and dorsal to 47/12 on the lateral IC. The top diagrams show lateral and ventral views of the intended lesion in the first experiment. The lesion was conservative and restricted to 121 , where cells active in working memory tasks have been found. The posterior limit of the lesions was defined by a line drawn between the anterior tips of the superior and inferior rami of the arcuate sulcus ( $A s$ and $A i$, respectively). Dorsally and ventrally the lesion was bounded by the principal sulcus $(S p)$ and ventral limit of the IC, respectively. The bottom diagrams show the intended lesion in the second experiment. The lesions extended further posteriorly to include area $45 \mathrm{~A}$ and further ventromedially to include all of area 47/12. In this case the posterior limit of the lesion was defined by a line drawn between the posterior tip of sulcus principalis and the anterior tip of the inferior ramus of the arcuate sulcus. The ventral limit was the lateral orbital sulcus (posterior) and the medial orbital sulcus (anterior). The central and lateral sulci ( $C S$ and $L S$, respectively) are also shown. 
Laboratory Care (National Institutes of Health publication 86-23, revised 1985) were followed. The studies were carried out under project and personal licenses from the British Home Office.

\section{Apparatus}

All the experiments were conducted with stimuli presented on a monitor with a touch-sensitive screen controlled by an IBM computer. A $20 \times 28$ $\mathrm{cm}$ Microvitec touch-sensitive monitor was used in experiment 1 , tasks $1-3$, and in the first preoperative assessment in experiment 2 . Touches were registered when hand movements interrupted an infrared beam. A larger $41 \times 29 \mathrm{~cm}$ Elographics touch-sensitive monitor was used in the second preoperative testing assessment and the postoperative assessment in experiment 2. The Elographics monitor uses a pressure-sensitive mechanism to register screen touches. The animals were tested in a transport cage, $30 \mathrm{~cm}$ from the monitor.

\section{General procedure}

In the first experiment six rhesus monkeys were trained preoperatively on visual pattern association (task 1). Lateral area $47 / 12$ on the IC was then removed in three of the animals (Fig. 1), and their postoperative performance was compared with their preoperative performance and with the performance of the three controls retested after a similar interval. The two groups of monkeys were then compared on new learning of color matching to a sample (task 2). Task 2 allowed us to test whether the absence of impairment in task 1 was simply attributable to our use of a visual pattern association paradigm as opposed to the more conventional color-matching design. Finally, their performance was compared on the visual association task with longer delays (task 3 ).

The three control animals were then used in the second experiment. Their performance on color matching to a sample was assessed on two occasions 3 weeks apart. Larger lesions of the IC, including all of areas 47/12 and 45A (Fig. 1), were then made, and the animals were retested postoperatively 3 weeks later.

\section{Experiment 1}

Task 1: visual pattern association task. The animals initially learned a simultaneous version of the task. Each trial began with the presentation of one of two cues in the center of the monitor. The cues were either blue or yellow rectangles $5 \mathrm{~cm}$ across and $7 \mathrm{~cm}$ in height. Touching the cue triggered the presentation of two response shapes on either side of the cue, all of which remained on the screen until the monkey responded. Only two response shapes were used, and both consisted of distinct black and white line patterns the same sizes as the cue shapes. One response pattern consisted of a black cross, consisting of vertical and horizontal bars, inside a white circle. The other response pattern consisted of a white-outlined, black-filled ellipse superimposed on a white, vertical bar. There was a distance of $4.5 \mathrm{~cm}$ between the edge of the cue shape and response shape. The correct response was determined by the color of the cue. Correct responses were rewarded with delivery of Noyes pellets or peanuts. The intertrial interval was $4 \mathrm{sec}$, unless the animal made an error, in which case it was increased to $7 \mathrm{sec}$ as punishment. Touching the screen between trials was punished by doubling the intertrial interval. A rerun correction procedure was used such that the same cue was presented until the correct response was made. Response shapes were presented randomly on either side of the cue shape. Each monkey performed 100 rewarded trials every day. The monkeys were trained to a criterion of $90 \%$ correct responses for 2 consecutive days. The error score of each animal corresponded to the total error score including errors made on correction trials.

Subsequently the animals were trained to perform the task with a $0 \mathrm{sec}$ delay. The procedure was identical to the simultaneous presentation task, except that the cue shape did not remain on the screen while the response shapes were present. Pressing the cue shape caused it to disappear and the response shapes to reappear as before. The animals were trained to perform to a $90 \%$ correct criterion over 2 consecutive days of testing. The number of trials to reach the criterion on the simultaneous version of the task varied between $\sim 2000$ and $>10,000$.

The animals then learned versions of the task involving 1 and $2 \mathrm{sec}$ delays between cue offset and response shape onset. The rerun correction procedure was always used. The delayed visual association task proved very difficult for some of the animals to learn; it required between just $>400$ trials to $>12,000$ trials. The animals were therefore trained to a lower criterion of $4 \mathrm{~d}$ at $80 \%$ correct performance on the 1 and $2 \mathrm{sec}$ delay conditions.

Before surgery the animals were then retested at 0 and $2 \mathrm{sec}$ delays to criteria of $80 \%$ correct performance over 4 d. Area 47/12 was then removed bilaterally in three of the animals, whereas the other three animals, matched for performance, remained as controls. Three weeks after surgery the animals were retested on simultaneous and 0 and $2 \mathrm{sec}$ delay versions of the task. The same criteria were always used (simultaneous, $2 \mathrm{~d}$ at $90 \%$; $0 \mathrm{sec}, 4 \mathrm{~d}$ at $80 \% ; 2 \mathrm{sec}, 4 \mathrm{~d}$ at $80 \%$ ).

Task 2: color matching. All the animals were then taught to perform matching to a sample with color stimuli. The cue stimuli used were of two different colors not used in task 1 , red and blue. The stimuli were the same sizes as those in task 1 . In this task the response shapes were also red and blue bars of the same sizes and shapes as the stimulus shapes. The procedure followed the same format as task 1 . A cue shape was presented on the center of the screen until touched. Subsequently, response shapes were presented on either side of the cue shape until the monkey responded. The response shapes were each of the two color bars. The correct response was to press the response shape identical in color to the cue shape. The stimuli were the same sizes and shapes as in task 1. The animals were first taught to perform simultaneous matching to a sample (SMS) and then subsequently proceeded to 0 and $2 \mathrm{sec}$ delayed match (DMS) versions. In the DMS versions the cue shape was extinguished as soon as it was touched and before the response shapes were presented. A criterion of $80 \%$ correct performance was used for the simultaneous version and 0 and $2 \mathrm{sec}$ delays. As before, the error score of an animal corresponded to the total error score including errors made on correction trials.

Task 3: visual pattern association task with long delays. Finally all the animals relearned the visual association task with longer delays of up to $8 \mathrm{sec}$. Animals were retrained to the same criteria on simultaneous and 0 and $2 \mathrm{sec}$ delay versions of the task (simultaneous, $2 \mathrm{~d}$ at $90 \% ; 0 \mathrm{sec}, 4 \mathrm{~d}$ at $80 \% ; 2 \mathrm{sec}, 4 \mathrm{~d}$ at $80 \%$ ). Again error scores corresponded to the total error scores including errors made on correction trials. A performance test was then carried out at 4,6, and 8 sec delays. Performance was tested by giving each animal $4 \mathrm{~d}$ of testing at each delay period. The animals then proceeded to the next stage of testing regardless of the level of their performance. The stimuli used in this experiment were slightly smaller than previously; the cue and response shapes were $2.5 \times 3.4 \mathrm{~cm}$ and, consequently, farther apart ( $8 \mathrm{~cm}$ edge to edge).

\section{Experiment 2: color matching}

The three animals that had served as controls took part in the second experiment. Preoperatively the animals were trained to perform color matching with delays of up to $8 \mathrm{sec}$. The colors were the same as those used in experiment 1 , task 2 . The stimulus size was the same as that used in experiment 1 , task 3 . Animals were trained to a $90 \%$ criterion $(2 \mathrm{~d})$ for SMS and then to an $80 \%$ criterion $(4 \mathrm{~d})$ in a $0 \mathrm{sec}$ delay condition and a $2 \mathrm{sec}$ DMS. The error score of each animal corresponded to the total error score including errors made on correction trials. This was followed by performance tests of $4 \mathrm{~d}$ at each delay period of 4,6 , and $8 \mathrm{sec}$. Finally there was another identical preoperative retention test after 3-4 weeks. Areas $47 / 12$ and $45 \mathrm{~A}$ were then removed bilaterally in all three animals. After a recovery period of 3-4 weeks the animals were retested again in an identical fashion.

\section{Surgery and histology.}

All surgery was carried out under sterile conditions with sodium pentobarbitone anesthesia and with the aid of a binocular operating microscope. Tissue was retracted in anatomical layers, and a bone flap was removed. The lesions were made by aspiration with a fine gauge sucker. The wound was closed in anatomical layers. At least 3 weeks were allowed for recovery before testing resumed.

When the animals had completed their testing they were anesthetized with sodium pentobarbitone and perfused with $90 \%$ saline and $10 \%$ formalin. The head was placed in a stereotaxic apparatus, and a vertical cut was made caudal to the lunate sulcus. The brains were then removed, photographed, and placed in $10 \%$ sucrose formalin until they sank. The brain was then cut at the position of the anterior commissure and cut in $50 \mu \mathrm{m}$ coronal sections. Every fifth section was stained with cresyl violet for analysis.

Experiment 1: removal of area 47/12. As explained in the introductory remarks, the lesions in the first experiment were deliberately conservative. The intention was to include area 47/12l (Petrides and Pandya, 1994) or 121 (Carmicheal and Price, 1994). Lateral and ventral views of the intended lesion are shown in Figure 1, top. Dorsally the lesion should extend to $5 \mathrm{~mm}$ below the sulcus principalis to the lateral orbital sulcus in the ventral direction. The posterior limit was defined as a line drawn between the most anterior points on the superior and inferior rami of the arcuate sulcus. 

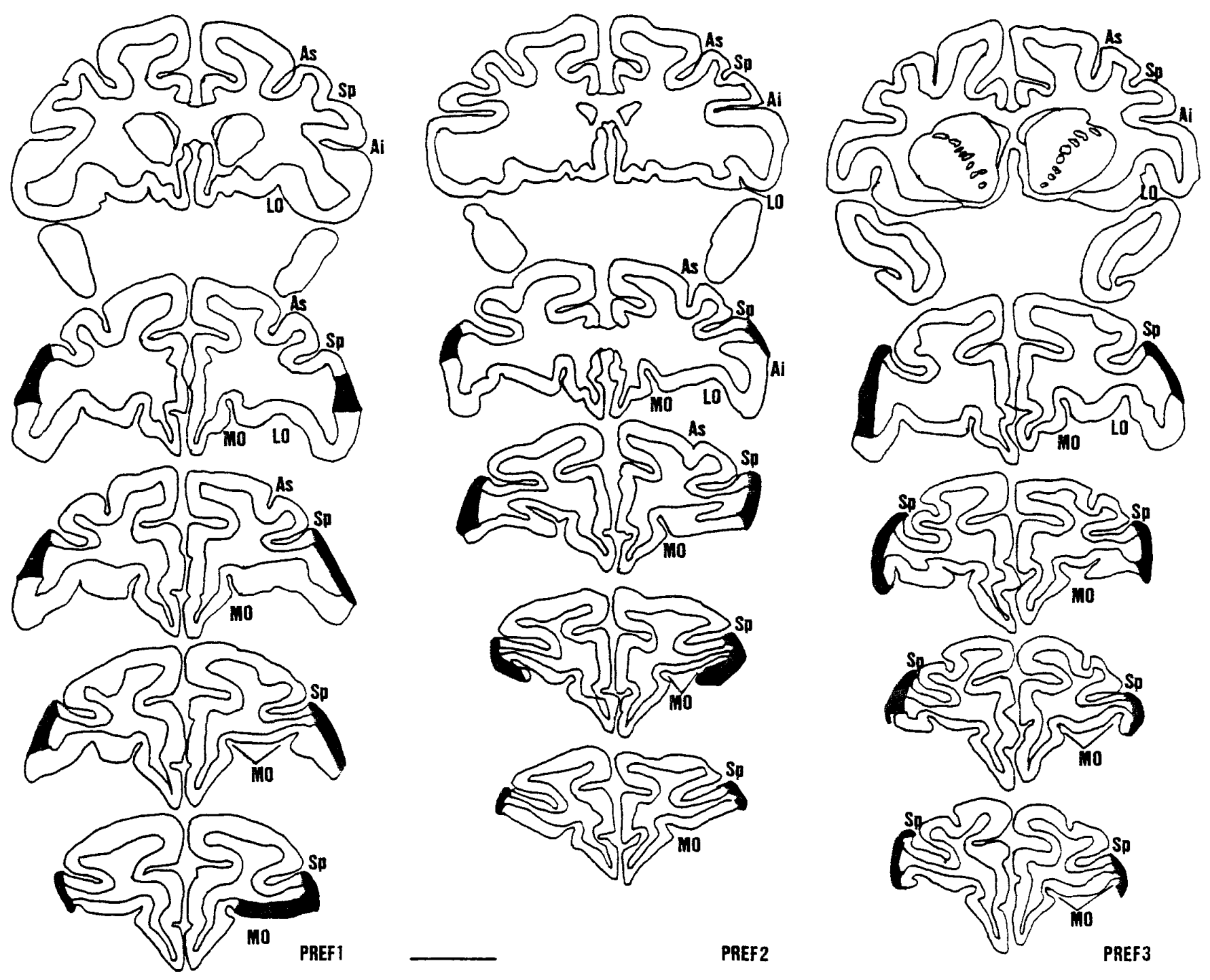

Figure 2. Coronal cross-sections through the prefrontal cortex in animals PREF1, PREF2, and PREF3 in experiment 1 . The most posterior section (top) is taken approximately 1-2 $\mathrm{mm}$ in front of the posterior tip of sulcus principalis $(S p)$. The lesion (indicated by the thick dark line joining areas of intact cortex) did not extend as far back as this section in any of the animals. The next section (second from top) is taken rostral to the last section to show the inferior ramus of the arcuate sulcus $(A i)$. The lesion can be seen in each animal at this level, but it is limited in extent. Its ventral limit is far from the lateral orbital sulcus $(L O)$. The third and fourth sections from top are taken at distances one-third and two-thirds between the second section and the most anterior section (bottom) taken where the lateral ramus of the bifurcated medial orbital sulcus $(M O)$ approaches the lateral surface. The superior ramus of the arcuate sulcus $(A s)$ is also shown. Scale bar, $1 \mathrm{~cm}$.

Figure 2 shows the lesions for experiment 1 . In all cases the lesions removed the lateral part of the convexity, area 47/12l. In every case there was incomplete damage to the orbital part of area $47 / 12$, because the lesions did not extend as far as the lateral orbital sulcus (47/12o, Petrides and Pandya, 1994; or 12o, Carmicheal and Price, 1994). This was particularly noticeable in the more posterior sections when the position of the lateral orbital sulcus was still relatively medial (for example, the second section shown for each animal in Fig. 2). The most posterior section (Fig. 2 , top row) for each animal shows that the tissue in the posterior inferior convexity, between the sulcus principalis and the inferior limb of the arcuate sulcus, remained intact in each case. One monkey, PREF3, sustained additional damage to anterior orbital area 11 bilaterally (see the most anterior section for PREF3 in Fig. 2).

Experiment 2: removal of areas $47 / 12$ and $45 \mathrm{~A}$. The lesions were similar in the second experiment, but the intention was to include area 45A as well as area 47/121 (Petrides and Pandya, 1994) on the lateral surface and $47 / 120$ on the orbital surface (see Fig. 1, center and bottom). The posterior limit of the lesion was defined by a line drawn from the back of the sulcus principalis to the most anterior point on the inferior ramus of the arcuate sulcus. We took care to avoid the tissue immediately adjacent to the arcuate sulcus (45B and area 8; Petrides and Pandya, 1994). In its more posterior part, the ventromedial extent of the lesion was defined by the lateral bank of the lateral orbital sulcus. More anteriorly the ventromedial extent of the lesion was defined by the lateral bank of the lateral bifurcation of the medial orbital sulcus (see Fig. 1, center and bottom).

Figure 3 shows the lesions for experiment 2 . The most posterior section for each animal (Fig. 3, top row) shows that the lesion extended more posteriorly in experiment 2 than it had in experiment 1 (for comparison, see the most posterior sections in Fig. 2, top row). In each case the lesion extended to the posterior end of the sulcus principalis. The second section for each animal was taken from approximately the level of the anterior tip of the inferior arcuate sulcus and shows large lesions in the lateral IC and the ventral surface as far as the lateral orbital sulcus (for comparison, see the second sections in Fig. 2). More anteriorly, in animals PREF4 and PREF5, the lesion extends as far as the lateral branch of the medial orbital sulcus. The lesion in PREF6 was smaller than in the other two animals, not quite reaching the lateral bank of the lateral orbital sulcus on the right. In PREF4 and PREF5 there was slight damage to the tissue just dorsal to the sulcus principalis at the back. 


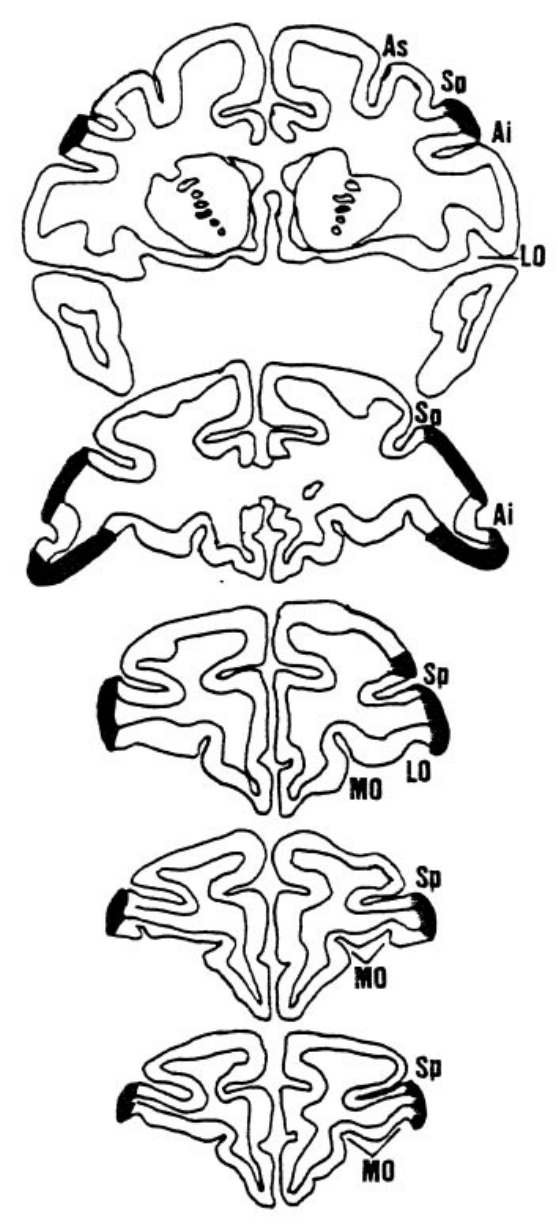

PREF4
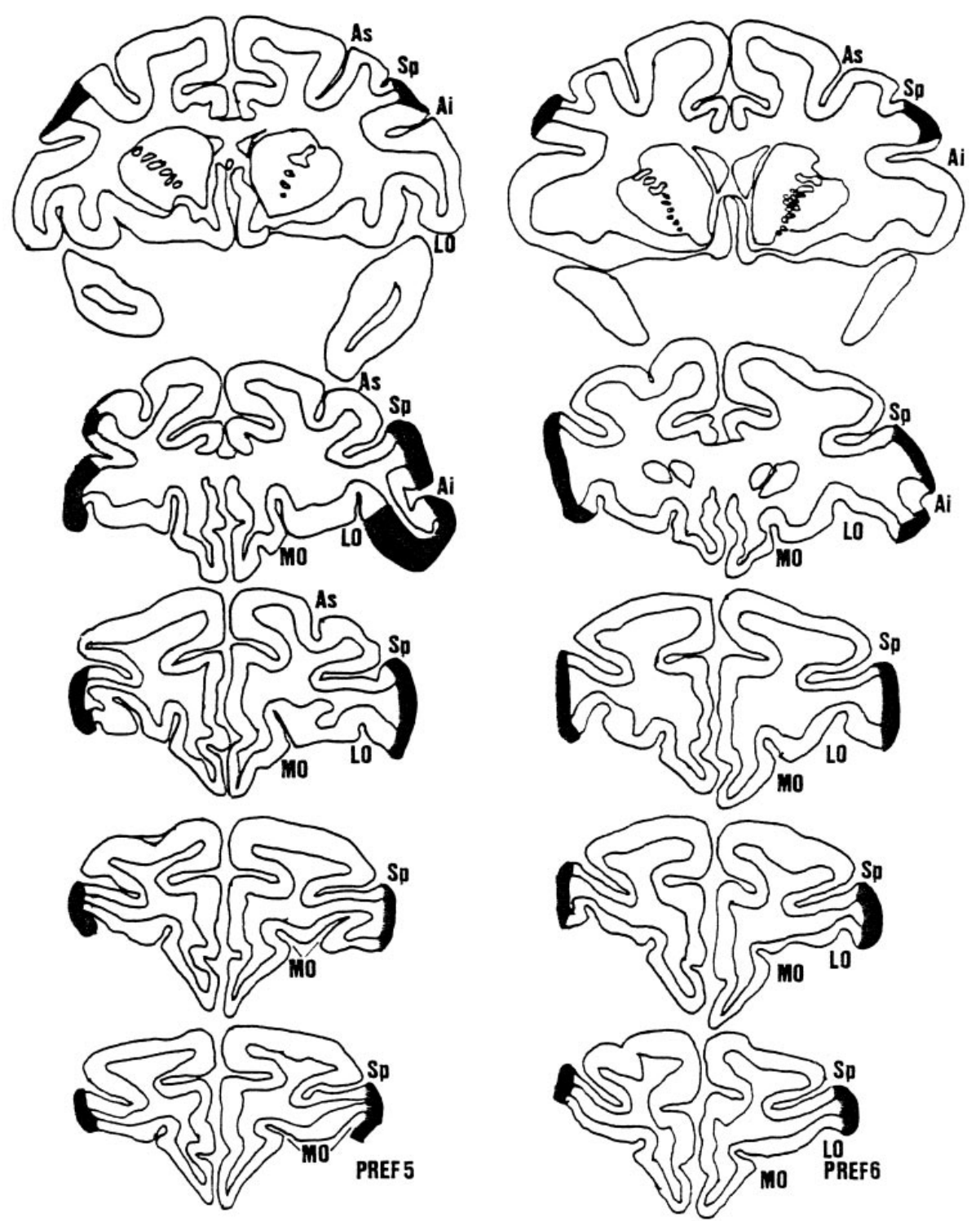

Figure 3. Coronal cross-sections through the prefrontal cortex in animals PREF4, PREF5, and PREF6 in experiment 2. The five sections are taken at approximately the same or slightly more posterior levels as those shown in Fig. 2. The most posterior section (top) is taken approximately $1 \mathrm{~mm}$ in front of the posterior tip of sulcus principalis $(S p)$ (there is some slight skew from the coronal plane in PREF4 and PREF5). The lesion is already present in this section in each case (compare Fig. 2, top, where there is no damage at this level). The next section (second from top) was taken at a level to include the anterior tip of the inferior arcuate sulcus $(A i)$ in at least one hemisphere. The lesion is extensive at this point and already extends toward the lateral orbital sulcus $(L O)$ on the ventral surface (compare Fig. 2, second from top, where the lesion even in a slightly more anterior section is limited to part of the lateral surface). Sections third and fourth from top are taken at a one-third and two-thirds of the distance between the second section and the most anterior section taken at the point where the lateral ramus of the medial orbital sulcus $(M O)$ approaches the lateral surface. The superior ramus of the arcuate sulcus $(A s)$ is also shown. Scale bar, $1 \mathrm{~cm}$ (same scale as in Fig. 2).

\section{Statistical comparisons}

Performance was assessed in two ways. Comparisons between groups were made using the nonparametric Mann-Whitney test. Where there were preoperative data, the preoperative and postoperative scores were listed and compared using a matched sample $t$ test.

\section{RESULTS}

\section{Experiment 1}

Task 1: visual pattern association task

The animals that underwent surgery were not impaired on the simultaneous task or the delayed tasks at 0 or 2 sec. Figure 4 shows the total error scores (including errors made on correction trials). Nonparametric group comparisons showed that, postoperatively, there was no significant difference between the performance of the two groups on simultaneous $(U=3.5 ; p=0.6579), 0$ sec delay $(U=$ $3.0 ; p=0.5127)$, or 2 sec delay $(U=3.0 ; p=0.5127)$ versions. When the comparisons were made between the preoperative and postoperative scores of each individual animal, it was apparent that in all cases the animals improved after the lesion. The improvement was significant for PREF1 and PREF3 at the $0 \mathrm{sec}$ delay $(t=4.80$; $\mathrm{df}=$ $3 ; p=0.017$; and $t=10.23$; $\mathrm{df}=3 ; p=0.002$, respectively) but not at the $2 \mathrm{sec}$ delay $(t=1.04 ; \mathrm{df}=6 ; p=0.338$; and $t=1.54$; df $=6$; $p=0.174$, respectively). For PREF2 the improvement was not significant for delays of either $0 \sec (t=0.74$; $\mathrm{df}=3 ; p=0.513)$ or $2 \sec (t=1.87$; df $=7 ; p=0.103)$. 


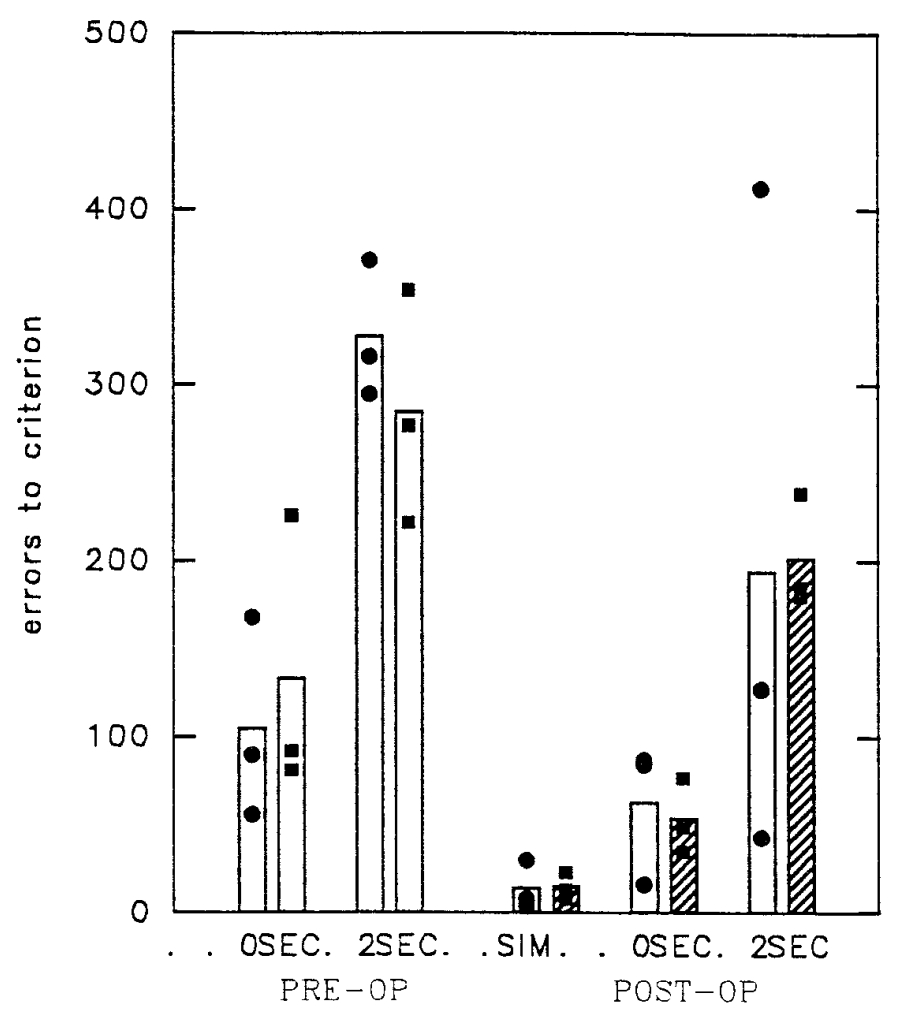

Figure 4. Visual associative task (experiment 1, task 1). The left side shows the total errors (including correction errors) each animal made in relearning to perform the visual association task with 0 and $2 \mathrm{sec}$ delays. A histogram bar is shown for the control group (always on the left) and the experimental group (always on the right) for each delay time. The right side shows the error scores for relearning the task with simultaneous presentation of the sample and match choices and with 0 and 2 sec delays. Hatching indicates postoperative performance of the experimental group after the ventral prefrontal cortex has been removed from area 47/12. Bar heights indicate group mean performance; $\boldsymbol{0}$ and $\mathbf{\square}$, performance of individual animals in the control and experimental groups, respectively.

\section{Task 2: color matching}

There was no evidence of any impairment on the color SMS or DMS in the animals that underwent surgery when their performance was compared with that of the control group. Figure 5 shows the total error scores (including errors made on correction trials). Group comparisons showed that there was no significant difference in performance in simultaneous $(U=3.0 ; p=0.5127)$, 0 sec delay $(U=3.0 ; p=0.5127)$, and $2 \sec$ delay $(U=3.0 ; p=$ 0.5127 ) versions of the task.

\section{Task 3: visual association task}

There was no evidence of any impairment in the performance of the animals that underwent surgery at even the longest delays when they were compared with the control group. Figure 6 shows the total number of errors (including errors made on correction trials) made in the first $4 \mathrm{~d}$ of testing for each of the delay periods. Group comparisons showed that there was no significant difference in performance at the $0 \mathrm{sec}$ delay $(U=4.0 ; p=0.8273), 2 \mathrm{sec}$ delay $(U=1.0 ; p=0.1266), 4$ sec delay $(U=2.0 ; p=0.2752), 6$ sec delay $(U=3.0 ; p=0.5127)$, or $8 \sec$ delay $(U=2.0 ; p=$ $0.2752)$.

It should be noted that the additional removal of area 11 in one animal, PREF3, did not have any detrimental effect on performance; PREF3 made fewer errors at every stage of all the tasks than either of the other two animals in the experimental group.

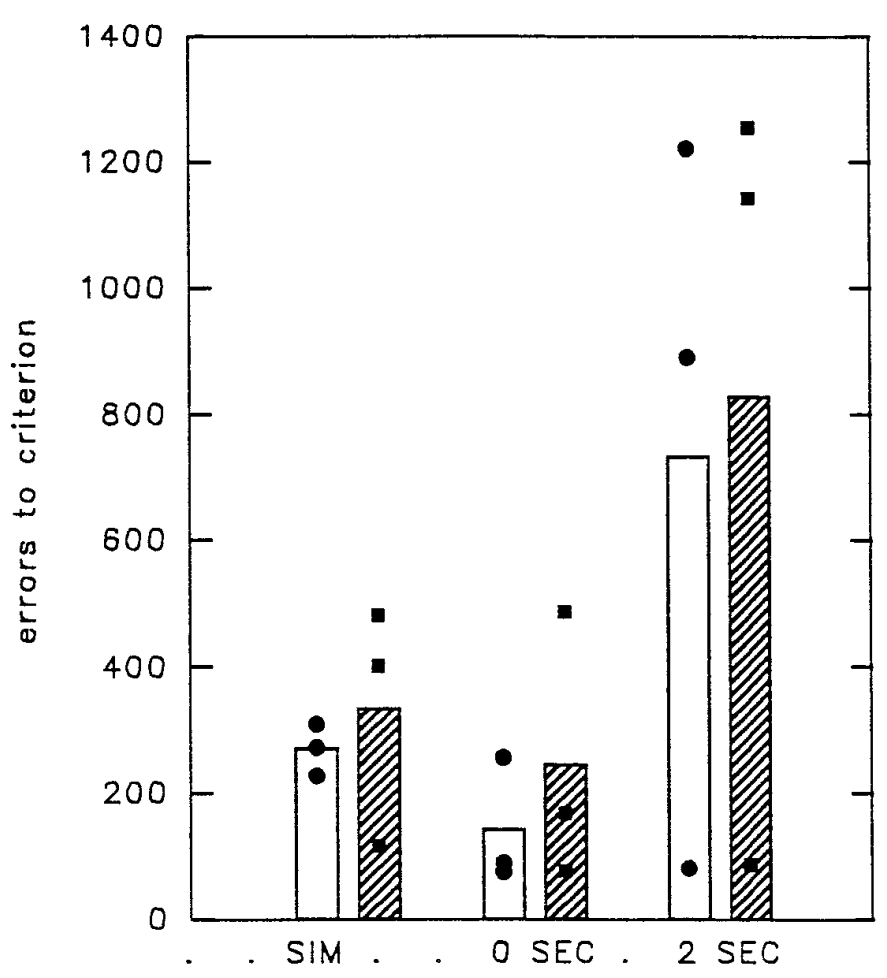

Figure 5. Color match (experiment 1, task 2). Shown is the total number of errors (including correction errors) made in the new, postoperative learning of the color matching task with simultaneous sample and match presentation and with delays of 0 and $2 \mathrm{sec}$. Hatching indicates the performance of the experimental group with a lesion in area 47/12. Bar heights indicate group mean performance; $\boldsymbol{0}$ and $\mathbf{\square}$, performance of individual animals in the control and experimental groups, respectively.

\section{Experiment 2}

Figure 7 shows each monkey's color SMS performance before and after surgery. In Figure 7 the total postoperative scores (including errors made on correction trials) are compared with the first preoperative scores. The second preoperative test scores are not shown, because there was a breakdown in the first touch screen and the animals had to adapt to using a second touch screen. The performance of the animals was disrupted while they learned how to ensure that their responses were registered. By the time they had performed the preoperative retention tests at 0 and $2 \mathrm{sec}$ immediately before surgery, all animals had adapted to the new monitor.

Postoperatively, all the animals made more errors during relearning of color SMS than they had made during the first preoperative test. The impairment was significant in each case, as assessed by a matched-sample $t$ test (PREF4: df $=1 ; t=93 ; p<$ 0.004; PREF5: df $=2 ; t=3.30 ; p<0.041$; PREF6: df $=4 ; t=$ $2.31 ; p<0.041)$.

Figure 8 shows the total number of errors (including errors in correction trials) made in obtaining the 400 rewards at each of the delay intervals. The data are shown for both preoperative tests, because preoperatively the animals had adapted to the second touch screen by the time they were given the second preoperative test.

The effect of the lesion was assessed by comparing the number of errors made in relearning the task with the criterion at 0 and 2 sec delays and the number of errors made during the attainment of 400 rewards ( $4 \mathrm{~d}$ of testing) on the performance testing at 0,2 , 4, 6, and $8 \mathrm{sec}$ delays. Postoperatively all the animals made more 


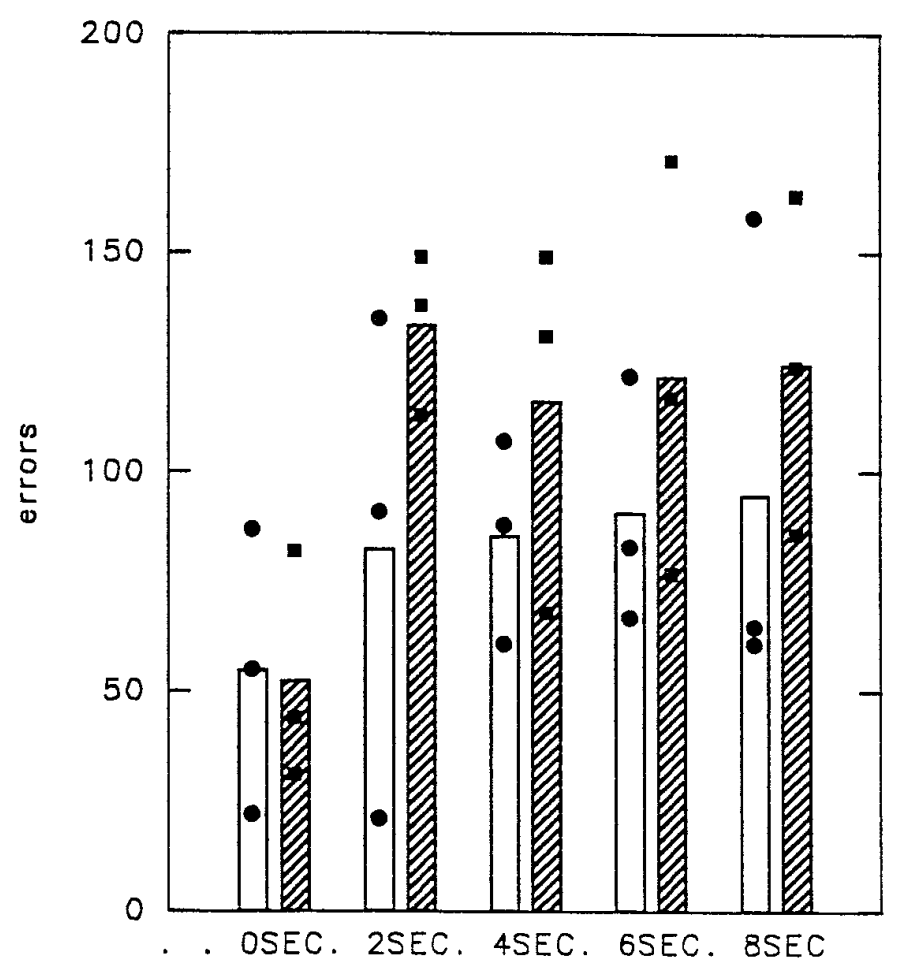

Figure 6. Visual association task (experiment 1, task 3). Shown is the total number of errors (including correction errors) made by each animal during the first 400 rewarded trials of the visual association task at each delay period $(0,2,4,6$, and $8 \mathrm{sec})$. Hatching denotes the experimental group with removal of area 47/12. Bar heights indicate group mean performance; and $\mathbf{\square}$, performance of individual animals in the control and experimental groups, respectively.

errors in relearning the task on 0 sec delay trials than they had during the second preoperative test. However, the impairment was only significant in PREF5, as assessed by a matched sample $t$ test $(\mathrm{df}=3 ; t=4.91 ; p=0.08)$. It was not significant for either of the other animals (PREF4: $\mathrm{df}=3 ; t=1.03 ; p=0.191$; PREF6: $\mathrm{df}=3 ; t=0.36 ; p=0.37)$, and as a group they performed similarly to the animals on the second occasion. All animals relearned the task in the minimum period of $4 \mathrm{~d}$ of testing as they had done before surgery.

There was no evidence of a delay-dependent deficit, because postoperative performance did not decline at longer delays (2, 4, 6 , and $8 \mathrm{sec}$ ). Postoperative group performance at the longer delays was always within the range of both the first and second preoperative test group performances. Although PREF5 always performed worse than on either preoperative test, PREF4 and PREF6 sometimes performed better and sometimes worse than before surgery.

\section{DISCUSSION}

\section{The anatomy of the inferior convexity}

We did not observe any delay-dependent defects after IC lesions. On the contrary, in the second experiment, SMS was profoundly impaired, but the imposition of delays, after initial relearning, caused no more difficulty than preoperatively (Figs. 7, 8).

It might be argued that the lesion was not selective enough to affect just DMS but leaving SMS intact. If the lesions were made any smaller, however, as in the first experiment, not only was there no deficit on any of the simultaneous presentation tasks, but there was no deficit on any of the delay trials either (Figs. 4-6).

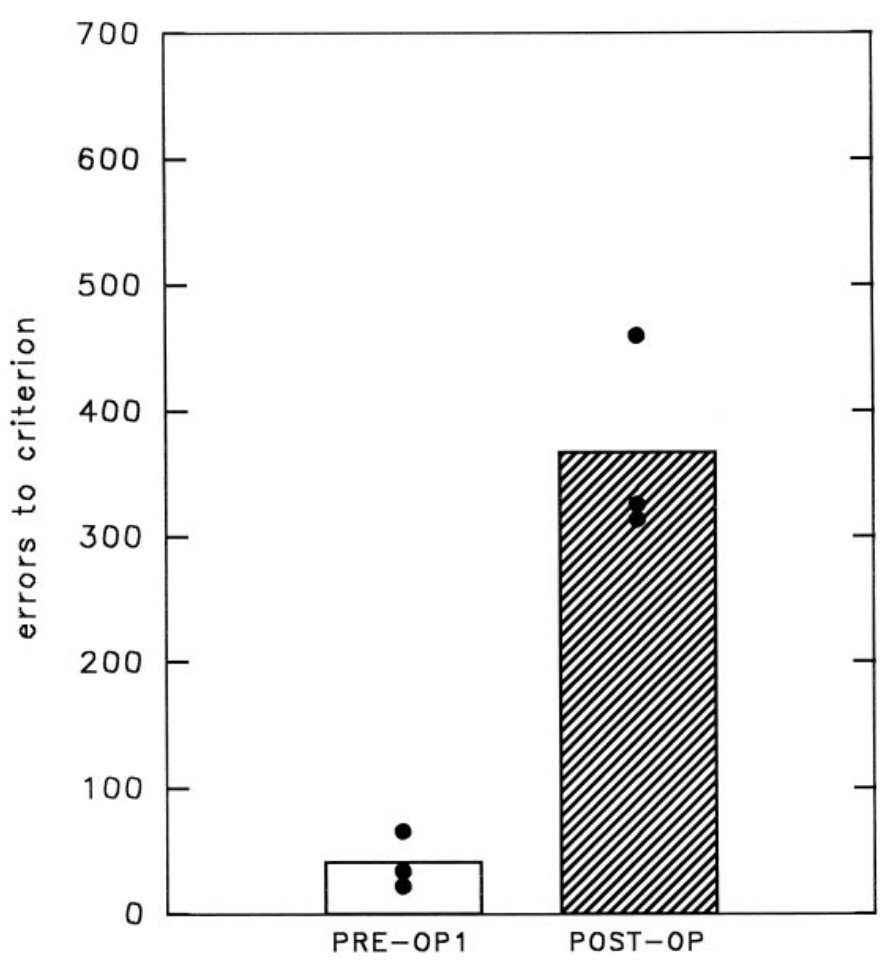

Figure 7. Color matching task (experiment 2). The column on the left indicates the total number of errors (including correction errors) during relearning of simultaneous color matching on the first preoperative retest. The hatched column on the right indicates the relearning scores after removal of ventral prefrontal cortex areas 47/12 and 45A. Bar heights indicate the group mean performance; $\boldsymbol{\bullet}$, performance of individual animals on each retest.

The small prefrontal lesions were made in the first experiment because we were concerned that previous reports of nondelay impairments (Iversen and Mishkin, 1970) might be attributable to damage to the prearcuate areas 45B and 8 (Petrides and Pandya, 1994). Removal of the prearcuate area in itself causes impaired performance on visual association tasks (Halsband and Passingham, 1982; Petrides 1985, 1987). The conservative lesions of lateral IC area 47/121 (Carmicheal and Price, 1994; Petrides and Pandya, 1994) in the first experiment were intended to avoid the prearcuate area but to remove the area in which cells have been reported with visual delay activity (Rosenkilde et al., 1981; Wilson et al., 1993; Miller et al., 1996). The larger lesions that did cause SMS impairments involved all of 47/12 (including 47/12o) as far as the lateral orbital sulcus and the more posterior $45 \mathrm{~A}$ and therefore included more of the tissue that is known to have visual projections (Webster et al., 1994). The inclusion of area 45A may have been critical in causing the deficit. There are also formrelated delay cells in anterior 45 (Goldman-Rakic, 1996), and retrograde dyes injected into this area also label most subdivisions of area TE and tissue just lateral and anterior to the rhinal sulcus (Pandya and Yeterian, 1996). The more ventromedial part of $47 / 12$ has connections with the superior temporal sulcus (Barbas, 1988) and contains visually responsive neurons that also respond to food rewards (Rolls and Baylis, 1994; Baylis et al., 1995).

\section{The inferior convexity and working memory}

According to Goldman-Rakic (1987, 1996), working memory guides behavior when sensory guidance is not possible. Studies have shown that sulcus principalis lesions disrupt memory-guided 


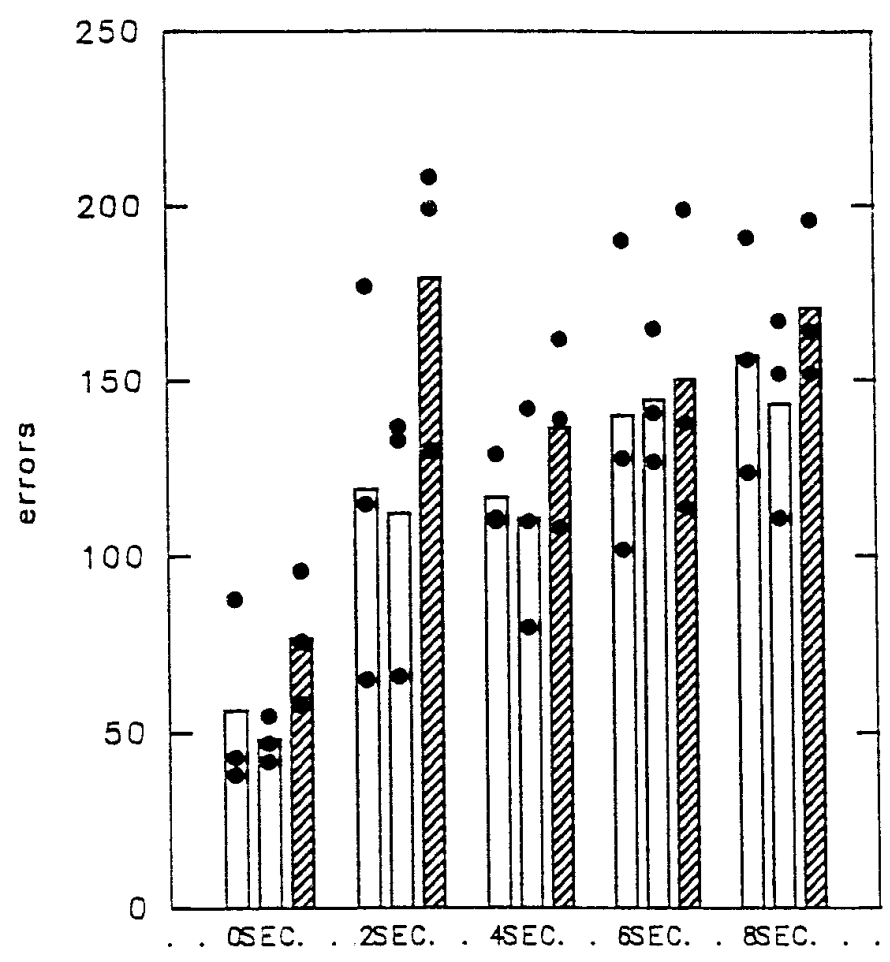

Figure 8. Color matching with delays (experiment 2). Shown is the total number of errors (including correction errors) made during the first 400 rewarded trials at each delay $(0,2,4,6$, and $8 \mathrm{sec})$ during the two preoperative tests and the postoperative test (hatched bar). Bar heights indicate group mean performance; $\boldsymbol{\bullet}$, performance of individual monkeys.

responses but not sensory-guided responses (Goldman and Rosvold, 1970; Passingham, 1985; Funahashi et al., 1993); these studies are consistent with the hypothesis that the sulcus principalis is involved in working memory. Funahashi et al. (1993) showed that even small principalis lesions impaired oculomotor, spatial DR with delays of $8 \mathrm{sec}$ but left a simultaneous version unaffected. The results of the present experiment were the reverse; IC lesions impaired relearning of SMS but not the subsequent performance of DMS with delays of up to $8 \mathrm{sec}$.

Our results suggest that previously reported deficits on DMS after IC lesions may actually reflect a problem in relearning the matching task, and that the delay may not have been crucial. Passingham (1975) started postoperative testing at $0 \mathrm{sec}$, and Mishkin and Manning (1978) started testing at $10 \mathrm{sec}$.

The results are consistent with previous reports of IC lesions causing impairments on nondelay tasks. Passingham (1975) also reported that monkeys with IC lesions were impaired on SMS after they had failed to relearn the task at $0 \mathrm{sec}$ delay. It could have been argued that prolonged experience of failure with $0 \mathrm{sec}$ delays led to the monkeys' acquiring a failure set that prevented them from learning SMS. The present results suggest that the impairment on SMS was genuine. Iversen and Mishkin (1970) also reported that monkeys with IC lesions were impaired on an auditory go-no go task without delay. The present results suggest that the inclusion of a response suppression element is not necessary for a task to be impaired by IC removal. Disconnection of the frontal and temporal lobes, either by uncinate fascicle section or crossed lesions, also causes impairment on pattern association tasks with no delay component (Eacott and Gaffan, 1992; Parker and Gaffan, 1997).

Kowalska et al. (1991) have previously reported that IC lesions impaired relearning of delayed nonmatching to a sample with trial unique objects with $10 \mathrm{sec}$ delays. Once the initial $10 \mathrm{sec}$ delay task was relearned, however, the monkeys were not impaired by lengthening the delays. The present finding of impaired SMS suggests that the animals in the study by Kowalska et al. (1991) were impaired at just relearning the nonmatching task, and even the $10 \mathrm{sec}$ delay component may not have been important. The absence of delay dependency in the impairment in the study of Kowalska et al. (1991) could have been explained by the use of trial unique stimuli; trial unique matching can be performed on the basis of recognition memory. The absence of DMS impairment in the present experiment cannot be explained in the same way. When just two color patterns are used, the one that constituted the incorrect response choice on one trial may be the correct choice on the subsequent trial; there is an opportunity for interference between trials, which should tax the ability of working memory to recall the relevant information on any given trial.

Furthermore, it should be noted that the impairments caused by IC lesions are not restricted to tasks with color or form stimuli. Spatial delayed response is also disrupted by IC lesions (Iversen and Mishkin, 1970; Passingham, 1975; Mishkin and Manning, 1978). This is consistent with the recent demonstration of parietal connections with IC (Cavada and Goldman-Rakic, 1989).

\section{Human ventral prefrontal cortex and working memory}

Parts of the human ventrolateral and dorsolateral prefrontal cortices (VLPFC and DLPFC, respectively) are homologous to the macaque IC and DLPFC (Petrides and Pandya, 1994; Rajkowska and Goldman-Rakic, 1995). It has been claimed that VLPFC has a modality-specific role in form and face working memory (Baker et al., 1996; Courtney et al., 1996; Goldman-Rakic, 1996; McCarthy et al., 1996). Although it is true that there is VLPFC activation during form and face DMS in these and other studies (Haxby et al., 1995; Swartz et al., 1995), there is similar activation in face, form, and color tasks that are performed in the absence of delay (Corbetta et al., 1991; Haxby et al., 1994; Kosslyn et al., 1994; McIntosh et al., 1996; Grady et al., 1996). This is shown graphically in Figure 9. The role of the human VLPFC, like the monkey IC, is not limited to tasks with a delay component.

There is evidence that a part of DLPFC is also important for nonspatial working memory tasks (Petrides et al., 1993; McCarthy et al., 1994). It is, in fact, activation in this region that distinguishes pattern DMS from SMS (Swartz et al., 1995) and increases with delay in face SMS (Haxby et al., 1995). The left ventral frontal tissue in opercular Broca's area, however, is also more active in delays (Haxby et al., 1995). It could be argued that there are two distinct populations of form and spatial cells within the DLPFC. If this is the case, however, the subareas cannot be resolved with current imaging methods.

The human VLPFC also resembles the monkey IC in that its role is not restricted to form, face, and color tasks; it is also concerned with spatial matching tasks whether they involve delays (Jonides et al., 1993; Anderson et al., 1994; Smith et al., 1995; Owen et al., 1996) or not (Corbetta et al., 1991). The activation of the VLPFC in spatial tasks is approximately $2 \mathrm{~cm}$ more posterior than the form, face, and color focus (Fig. 9B). One study that claimed VLPFC activation in form tasks but not in spatial tasks (McCarthy et al., 1996) just considered coronal slices taken at the level of the more anterior VLPFC form area.

\section{Conclusion}

In summary, the results suggest that the DLPFC and the IC are not parallel working memory mechanisms, with the DLPFC op- 

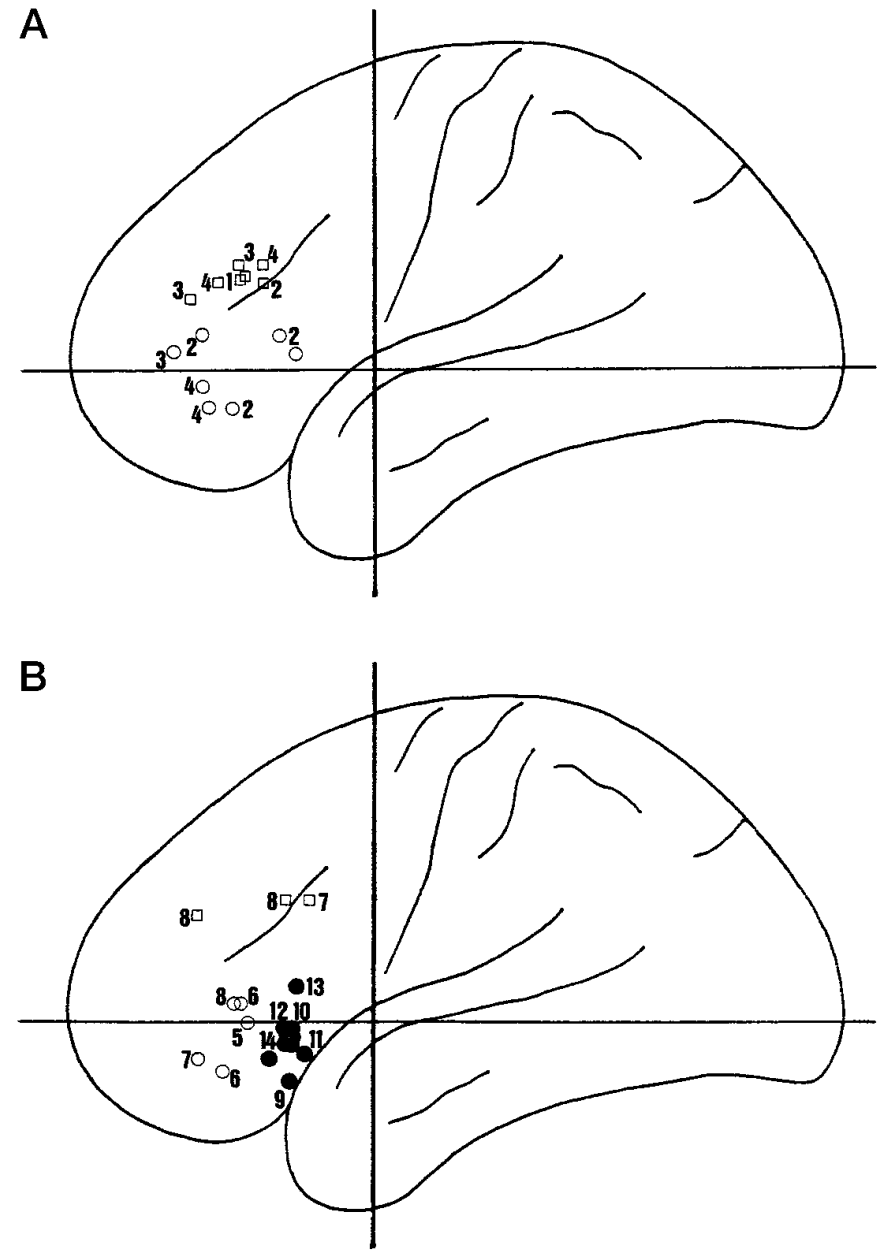

Figure 9. Peak regional cerebral blood flow changes in the human prefrontal cortex during matching tasks. Summarized are positron emission tomography studies in which the Tailarach and Tournoux (1988) coordinate system has been used. Peaks from both hemispheres are plotted onto the same figures. $A$, There are clear foci of activation in the VLPFC during form and face DMS and other working memory tasks $(\bigcirc)$. Other foci, however, clearly fall into the DLPFC ( $\square$ ). 1, From Petrides et al. (1993); 2, Haxby et al. (1995); 3, Baker et al. (1996); and 4, Courtney et al. (1996). The more posterior ventral foci lie at some distance from the rest and were recorded by Haxby et al. (1995); they may be in Broca's area in the left hemisphere. $B$, There are also similar VLPFC $(\bigcirc)$ and even some DLPFC $(\square)$ foci when subjects perform form, face, and color SMS tasks. 5, From Corbetta et al. (1991); 6, Haxby et al. (1994); 7, Kosslyn et al. (1994); and 8, Grady et al. (1996). Although it is clear that spatial working memory tasks activate DLPFC (not shown), the VLPFC is also frequently activated $(\bullet)$. Within the VLPFC, however, there seems to be an anteriorposterior division between nonspatial and spatial foci. Some of the spatial foci were recorded in SMS tasks (9, from Corbetta et al., 1991); others were recorded in DMS tasks (10, from Jonides et al., 1993; 11, Anderson et al., 1994; 12, Smith et al., 1995; 13, Baker et al., 1996; and 14, Owen et al., 1996).

erating on spatial input and the IC operating on visual inputs (Goldman-Rakic, 1987, 1996). The pattern of impairment after IC damage is not analogous to that seen after principalis damage. First the deficit does not depend on the imposition of a delay. Second, it does not depend on interference from non-trial unique stimuli. Third, both spatial and nonspatial tasks are impaired. Petrides (1994, 1996) has suggested an alternative "two-level" hypothesis of prefrontal function. The DLPFC is involved in second-order manipulation and monitoring of both spatial and nonspatial information in working memory. The IC is involved in "first order executive processes, such as active selection, comparison and judgement of stimuli held in short-term and long-term memory" (Petrides, 1996, page 1457). The present finding is consistent with the two-level hypothesis, because active selection, comparison, and judgment of the cue and response shapes are essential for SMS. Our findings, however, suggest that the role of the IC in selection, comparison, and judgment extends beyond those situations in which the stimuli must be held in memory.

\section{REFERENCES}

Anderson TJ, Jenkins IH, Brooks DJ, Hawken MB, Frackowiak RSJ, Kennard C (1994) Cortical control of saccades and fixation in man. A PET study. Brain 117:1073-1084.

Baker SC, Frith CD, Frackowiak RSJ, Dolan RJ (1996) Active representation of shape and spatial location in man. Cereb Cortex 6:612-619.

Barbas H (1988) Anatomic organization of the basoventral and mediodorsal visual recipient prefrontal regions in the rhesus monkey. J Comp Neurol 276:313-342.

Baylis LL, Rolls ET, Baylis GC (1995) Afferent connections of the caudolateral orbitofrontal cortex taste area of the primate. Neuroscience 64:801-812.

Butters N, Pandya D (1969) Retention of delayed alternation: effect of selective lesions of sulcus principalis. Science 165:1271-1273.

Carmicheal ST, Price JL (1994) Architectonic subdivision of the orbital and medial prefrontal cortex in the macaque monkey. J Comp Neurol 346:366-402.

Cavada C, Goldman-Rakic PS (1989) Posterior parietal cortex in rhesus monkey: II. Evidence for segregated corticocortical networks linking sensory and limbic areas with the frontal lobe. J Comp Neurol 287:422-445.

Corbetta M, Miezin FM, Dobmeyer S, Shulman GL, Petersen SE (1991) Selective and divided attention during visual discrimination of shape, color, and speed: functional anatomy by positron emission tomography. J Neurosci 11:2383-2402.

Courtney SM, Ungerleider LG, Keil K, Haxby JV (1996) Object and spatial visual working memory activate separate neural systems in human cortex. Cereb Cortex 6:39-49.

Deiber MP, Ibanez V, Sadato N, Hallet M (1996) Cerebral structures participating in motor preparation in humans: a positron emission tomography study. J Neurophysiol 75:233-247.

Eacott MJ, Gaffan D (1992) Inferotemporal-frontal disconnection: the uncinate fascicle and visual associative learning in monkeys. Eur J Neurosci 4:1320-1332.

Funahashi S, Kubota K (1994) Working memory and prefrontal cortex. Neurosci Res 21:1-11.

Funahashi S, Bruce CJ, Goldman-Rakic PS (1993) Dorsolateral prefrontal lesions and oculomotor delayed response performance: evidence for mnemonic "scotomas." Neuroscience 13:1479-1497.

Goldman PS, Rosvold HE (1970) Localization of function within the dorsolateral prefrontal cortex of the rhesus monkey. Exp Neurol 27:291-304.

Goldman-Rakic PS (1987) Circuitry of primate prefrontal cortex and regulation of behavior by representational memory. In: Handbook of physiology: the nervous system (Plum F, Mountcastle V, eds), pp 373-417. Bethesda, MD: American Physiological Society.

Goldman-Rakic P (1996) The prefrontal landscape: implications of functional architecture for understanding human mentation and the central executive. Philos Trans R Soc Lond [Biol] 351:1445-1453.

Grady CL, Horwitz B, Pietrini P, Mentis M, Ungerleider LG, Rapoport SI, Haxby JV (1996) The effect of task difficulty on cerebral blood flow during perceptual matching of faces. Hum Brain Mapp 4:227-240.

Halsband U, Passingham RE (1985) Premotor cortex and the conditions for movement in monkeys (Macaca fascicularis). Behav Brain Res 18:269-277.

Haxby JV, Horowitz B, Ungerleider LG, Maisog JM, Peitrini P, Grady CL (1994) The functional organization of human extrastriate cortex: a PET-rCBF study of selective attention to faces and locations. J Neurosci $14: 6336-6353$.

Haxby JV, Ungerleider LG, Horwitz B, Rapoport SI, Grady CL (1995) Hemispheric differences in neural systems for face working memory: a PET-rCBF study. Hum Brain Mapp 3:68-82.

Iversen SD, Mishkin M (1970) Perseverative interference in monkeys 
following selective lesions of the inferior prefrontal convexity. Exp Brain Res 11:376-386.

Jonides J, Smith EE, Koeppe RA, Awh E, Minoshima S, Mintun MA (1993) Spatial working memory in humans as revealed by PET. Nature 363:623-624.

Kalaska JF, Crammond DJ (1995) Deciding not to GO: neuronal correlates of response selection in a $\mathrm{GO} / \mathrm{NOGO}$ task in primate premotor and parietal cortex. Cereb Cortex 5:1047-3211.

Kosslyn SM, Alpert NM, Thompson WL, Chabris CF, Rauch SL, Anderson AK (1994) Identifying objects from different viewpoints. A PET investigation. Brain 117:1055-1071.

Kowalska DM, Bachevalier J, Mishkin M (1991) The role of the inferior prefrontal convexity in performance of delayed nonmatching-to-sample. Neuropsychologia 29:583-600.

McCarthy G, Blamire A, Puce A, Nobre A, Bloch G, Hyder F, GoldmanRakic P, Shulman RG (1994) Functional magnetic resonance imaging of human prefrontal cortex activation during a spatial working memory task. Proc Natl Acad Sci USA 91:8690-8694.

McCarthy G, Puce A, Constable RT, Krystal JH, Gore JC, GoldmanRakic P (1996) Activation of human prefrontal cortex during spatial and non-spatial working memory tasks measured by functional MRI. Cereb Cortex 6:600-613.

McIntosh AR, Grady CL, Haxby JV, Ungerleider LG, Horwitz B (1996) Changes in limbic and prefrontal functional interactions in a working memory task for faces. Cereb Cortex 6:571-584.

Miller EK, Erickson CA, Desimone R (1996) Neural mechanisms of visual working memory in prefrontal cortex of the macaque. J Neurosci 16:5154-5167.

Mishkin M (1957) Effects of small prefrontal lesions on delayed alternation in monkeys. J Neurophysiol 220:615-622.

Mishkin M, Manning FJ (1978) Non-spatial memory after selective prefrontal lesions in monkeys. Brain Res 143:313-323.

Owen AM, Evans AC, Petrides M (1996) Evidence for a two-stage model of spatial working memory processing within the lateral frontal cortex: a positron emission tomography study. Cereb Cortex 6:31-38.

Parker A, Gaffan D (1997) Frontal temporal disconnection in monkeys: dissociation between conditional and associative tasks and between unilateral and bilateral frontal lesions. J Neurosci, in press.

Pandya DN, Yeterian EH (1996) Comparison of prefrontal architecture and connections. Philos Trans R Soc Lond [Biol] 351:1433-1444.

Passingham RE (1975) Delayed matching after selective prefrontal lesions in monkeys. Brain Res 92:89-102.

Passingham RE (1985) Memory of monkeys (Macaca mulatta) with lesion in prefrontal cortex. Behav Neurosci 99:2-21.
Passingham RE (1988) Premotor cortex and preparation for movement. Exp Brain Res 70:590-596.

Passingham RE (1993) Oxford psychology series, 21: the frontal lobes and voluntary action. Oxford: Oxford UP.

Petrides M (1985) Deficits in nonspatial conditional associative learning after periarcuate lesions in the monkey. Behav Brain Res 16:95-101.

Petrides M (1987) Conditional learning and primate frontal lobes. In: The frontal lobes revisited (Perecman E, ed), pp 91-108. New York: Institute for Research in Behavioral Neuroscience.

Petrides M (1996) Specialized systems for the processing of mnemonic information within the primate frontal cortex. Philos Trans R Soc Lond [Biol] 351:1445-1453.

Petrides M, Pandya DN (1994) Comparative architectonic analysis of the human and the macaque frontal cortex. In: Handbook of neuropsychology, Vol 9 (Boller F, Grafman J, eds), pp 17-58. Amsterdam: Elsevier.

Petrides M, Alivasatos B, Evans AC, Meyer E (1993) Dissociation of human mid-dorsolateral from posterior dorsolateral frontal cortex in memory processing. Proc Natl Acad Sci USA 90:873-877.

Rajkowska G, Goldman-Rakic PS (1995) Cytoarchitectonic definition of prefrontal areas in the normal human cortex: II. Variability in locations of areas 9 and 46 and relationship to the Tailarach coordinate system. Cereb Cortex 5:323-337.

Rolls ET, Baylis LL (1994) Gustatory, olfactory, and visual convergence within the primate orbitofrontal cortex. J Neurosci 14:5437-5452.

Rosenkilde CE, Bauer RH, Fuster JM (1981) Single cell activity in ventral prefrontal cortex of behaving monkeys. Brain Res 209:375-394.

Smith EE, Jonides J, Koeppe RA, Awh E, Schumacher EH, Minoshima S (1995) Spatial versus object working memory: PET investigations. J Cognit Neurosci 7:337-356.

Swartz BE, Halgren E, Fuster JM, Simpkins F, Gee M, Mandelkern M (1995) Cortical metabolic activation in humans during a visual memory task. Cereb Cortex 3:205-214.

Webster MJ, Bachevalier J, Ungerleider LG (1994) Connections of inferior temporal areas TEO and TE with parietal and frontal cortex in macaque monkeys. Cereb Cortex 5:470-483.

Wilson FAW, Scalaidhe SPO, Goldman-Rakic PS (1993) Dissociation of object and spatial processing domains in primate prefrontal cortex. Science 260:1955-1957.

Wise SP, Mauritz KH (1985) Set related neuronal activity in the premotor cortex of rhesus monkeys: effects of changes in motor set. Proc R Soc Lond [Biol] 223:331-354. 